\title{
Dexmedetomidine versus ketamine in attenuating neuropathic pain through modulating STING/TBK pathway to modulate spinal ER-phagy in neuropathic pain model
}

\section{Yongda Liu}

Shengjing Hospital of China Medical University https://orcid.org/0000-0002-1544-9455

\section{Mengmeng Ding}

Shengjing Hospital of China Medical University

\section{Zhibin Wang}

Shengjing Hospital of China Medical University

\section{Xingyue Li}

Shengjing Hospital of China Medical University

Jiao Guo

Shengjing Hospital of China Medical University

\section{Guang Han}

Shengjing Hospital of China Medical University

Ping Zhao ( $\nabla$ zhaoping_sj@163.com)

Shengjing Hospital of China Medical University https://orcid.org/0000-0001-6392-5391

\section{Research article}

Keywords: neuropathic pain, endoplasmic reticulum stress, autophagy, ketamine, dexmedetomidine, STING pathway

Posted Date: March 23rd, 2020

DOI: https://doi.org/10.21203/rs.3.rs-18652/v1

License: (9) This work is licensed under a Creative Commons Attribution 4.0 International License. Read Full License 


\section{Abstract}

Background: Our previous studies suggested that stimulator of interferon genes (STING) level was altered in medial prefrontal cortex (MPFC) of spinal nerve ligation (SNL) rats.

Methods: In this study, we investigated that whether dexmedetomidine and ketamine provide antianxiety and anti-nociceptive effects via modulating spinal STING/TBK pathway to alter ER-phagy in SNL rats. We evaluated the analgesia and antianxiety effects of ketamine and dexmedetomidine in SNL rats. 2'3'cGAMP (STING pathway agonist) was administrated to investigate whether enhanced spinal STING pathway could reverse dexmedetomidine or ketamine treatment effects in SNL rats respectively. Analgesia effects were measured with mechanical withdrawal threshold (MWT) and antianxiety effect was measured with open field test (OFT). Proteins expression levels were evaluated by Western blotting. Distribution and cellular localization of STING pathway and ER stress were evaluated by confocal immunofluorescence.

Results: SNL induced mechanical hypersensitivity and anxiety; STING pathway was involved in the modulation of ER stress and ER-phagy in SNL rats; Dexmedetomidine and ketamine provided analgesia and antianxiety effects in SNL rats; Dexmedetomidine and ketamine alleviated ER stress via inhibiting STING pathway to enhance ER-phagy in SNL rats.

Conclusions: In all, both ketamine and dexmedetomidine provided antianxiety and anti-nociceptive effects through alleviating ER stress via inhibiting STING/TBK pathway to modulate spinal ER-phagy in SNL rats.

\section{Background}

There is no denying that neuropathic pain could be a refractory disease in clinic, which resulted from nervous system dysfunction caused by nerve system damage $(1,2)$. The hyper-excitability and neuroplasticity may result from diverse cellular and molecular alterations in the spinal cord following peripheral nerve injury, which play an essential role in the onset and maintenance of neuropathic pain(3). Treatment for neuropathic pain is a crucial challenge in clinic because the mechanisms underlying remain far from clear and pharmacotherapy treatments available might be ineffective(4). Therefore, the combination and adjuvant pharmacotherapy for neuropathic pain demand prompt solution.

Dexmedetomidine, a selective a2 adrenergic receptor (AR) agonist, provided analgesic potency via systemic administration, also, low does of dexmedetomidine relieved tactile allodynia induced by neuropathic pain model(5). Moreover, study demonstrated that dexmedetomidine might a better adjuvant for clinical procedure and pain management for neuropathic pain in clinic $(6,7)$.

Studies revealed that low does and antidepressant does level of ketamine, a sedative agent, might be a promising treatment for neuropathic pain(8). Ketamine administration showed positive analgesic and anti-nociceptive results for neuropathic conditions in animal models and in clinical patients $(8,9)$. 
Neuropathic pain led to endoplasmic reticulum (ER) stress and autophagy impairment(10, 11). Autophagy served as a protective effect and attenuated neuropathic pain via restoring homeostasis in spinal cord $(12,13)$. Autophagy following ER stress mainly induced ER-phagy which could selective degrade ER via autophagy processing(14-18). Thus, ER-phagy might be a promising treatment target for neuropathic pain.

Stimulator of interferon genes (STING), an ER-resident protein, provided a novel crosstalk between ER stress and ER-phagy(19-21). STING serves as an ER adaptor, being required in elevated ER stress condition(22). Its conformational changes and phosphorylation result in its translocation from ER to distinct perinuclear endosomes near Golgi(20, 23, 24). STING phosphorylation induces TANK binding kinase 1 (TBK1) phosphorylation and subsequently leads to the phosphorylation of interferon regulatory factor3 (IRF3) production $(25,26)$.

Our previous studies suggested that ER-phagy was altered in neuropathic pain model $(27,28)$. In this study, our aim was to investigate the effects of dexmedetomidine and ketamine administration on anxiety- and nociceptive-related behaviors; investigate whether dexmedetomidine and ketamine alter spinal ER-phagy in SNL rats via modulating STING/TBK pathway.

\section{Methods}

\section{Animals}

Male Sprague Dawley (SD) rats $(180-230 \mathrm{~g})$ were obtained from the Changsheng biological company and housed in Shengjing Hospital Benxi experiment base. This study was in accordance with the ethical guidelines of China Medical University for the use of laboratory animals and approved by the Animal Ethics Care and Use Committee of China Medical University Shengjing Hospital (approved number区 2016PS013K). All surgery was performed under $1 \%$ pentobarbital sodium anesthesia ( $35 \mathrm{mg} / \mathrm{kg}$ i.p.). Rats were randomly separated into different groups. $n=6$ rats per group. Timeline of experimental procedure was demonstrated as Figure 1.

\section{Spinal nerve ligation (SNL)}

Spinal nerve ligation procedure was carried out as described previously(29). Rats were placed in the prone position under anesthesia. A $2 \mathrm{~cm}$ incision was made on the left lumber 5 level, $0.5 \mathrm{~cm}$ approximately to midline. The left L5 and L6 spinal nerve were separated and ligated tightly with 4-0 silk thread, distal end of ligation was transected.

\section{Behavior tests}

\section{Mechanical withdrawal threshold (MWT)}

To examine the mechanical hypersensitivity of rats, the mechanical withdrawal threshold (MWT) test was measured with Von Frey filaments (Stoelting Company, Wood Dale, IL, USA) as demonstrated 
previously $(10,30)$. Rats were habituated 30 min before MWT test in plexiglass chamber. A positive withdrawal threshold of the hind paw was measured with up and down procedure. For each MWT trial, stimulation duration was 5 s approximately; interval time was 5 min; cut-off value was $15 \mathrm{~g}$.

\section{Open field test (OFT)}

The anxiety and locomotor level was evaluated with open field test as reported previously(31). The open field arena consisted of an aluminum plate base $(100 \mathrm{~cm} \times 100 \mathrm{~cm})$ surrounded by walls of $45 \mathrm{~cm}$. The interior was painted black. Open field arena was equipped with infrared detectors and analyzed by Noldus software. Rats were put in open field for $10 \mathrm{~min}$. The distance, the proportion of the path in the center, travel trace and heat map were demonstrated. The field was cleaned with $75 \%$ ethanol after each trial.

\section{Drug delivery}

$5 \mu \mathrm{g}$ 2'3'-cGAMP (STING agonist, Cat: \#tIrl-nacga23, InvivoGen, USA) was intrathecal injected with $5 \mu$ l Hamilton microsyringe at $4 \mathrm{~h}$ before operation and postoperative day $2,4,6$. Single intrathecal injections were administrated via percutaneous lumbar puncture through 5 th or 6 th intervertebral space. A rapid tail flick manifested that the microsyringe penetrated the dura mater. The injection speed was $0.5 \mathrm{~s} / \mu \mathrm{l}$ approximately and microsyringe was hold still for $30 \mathrm{~s}$ after injection.

Ketamine (20 mg/kg, Cat. \#1709291, Fujian Gutian Pharmaceutical Co., Ltd. China) or vehicle was intraperitoneally (i.p.) injected at $4 \mathrm{~h}$ before operation and postoperative day 2, 4, 6 .

Dexmedetomidine (20 $\mu \mathrm{g} / \mathrm{kg}$, Cat. \#181017BP, Jiangsu Hengrui Pharmaceutical Co., Ltd. China) or vehicle was intraperitoneally (i.p.) injected at $4 \mathrm{~h}$ before operation and postoperative day 2, 4, 6 .

\section{Western blot}

At postoperative day 7, SD rats were deeply anaesthetized and sacrificed. The L4 L6 spinal cord were rapidly dissected and frozen at $-80^{\circ} \mathrm{C}$. The tissues were homogenized in RIPA buffer (p0013B, Beyotime, China) and phosphatase inhibitors (1:100, Solarbio, China) for 30 min on ice, then centrifugation at $14,000 \mathrm{rpm}$ for $40 \mathrm{~min}$ at $4{ }^{\circ} \mathrm{C}$. The supernatant fraction after centrifugation was collected. The lysate with loading buffer (Beyotime, China) were separated with 12\% SDS/PAGE gel and transferred to PVDF membranes (GE, USA). Primary antibodies were incubated at $4{ }^{\circ} \mathrm{C}$ overnight (12h at least) respectively after $1 \mathrm{~h}$ room temperature blocking in 5\% BSA with TBST ( $0.1 \%$ Tween 20 in Tris-buffered saline). Bands were incubated with HRP-conjugated second antibodies for $1.5 \mathrm{~h}$ at room temperature. After 3 times TBST washing, signal was detected with ECL plus kit (Tanon, China bands), captured with the chemiluminescence imaging systems (GE, USA; c300, Azure biosystems, USA) and quantified with Image J software (NIH, USA).

The following antibodies were used in this study: rabbit Grp78 (1:2000, Abcam, USA), rabbit LC3 (1:1000, CST, USA;), rabbit p62 (1:2000, CST, USA), rabbit FAM134B (1:1000, Abcam, USA), rabbit p-STING (1:1000, CST, USA), rabbit STING (1:1000, CST, USA), rabbit p-TBK (1:1000, CST, USA), TBK (1:1000, CST, USA), 
mouse GAPDH (1:8000, Solarbio, China), goat anti-rabbit/goat anti-mouse IgG horseradish peroxidase (1:5000, Beyotime, China).

\section{Immunofluorescence staining}

Rats were deeply anesthetized and perfused with $0.9 \% \mathrm{NaCl}$ solution transcardially, followed by cold $4 \%$ paraformaldehyde in 0.1M PBS. L5 spinal cords were removed, post-fixed in fixative solution for $24 \mathrm{~h}$, and dehydrated with $30 \%$ sucrose in $\mathrm{ddH} 2 \mathrm{O}$ at $4^{\circ} \mathrm{C}$ for $24 \mathrm{~h}$. The brains were embedded with optimal cutting temperature (OCT, SAKURA, USA) compound. Embedded L 5 tissue was coronal sectioned in cryostat at $10 \mu \mathrm{m}$ thickness. For confocal immunostaining, sections were incubated with anti-NeuN (neuronal marker, 1:200, MAB377, Millipore, USA)/GFAP (glial cell marker, 1:200, Abcam, China) and anti-Grp78 (ER stress marker, 1:200, Abcam, USA)/p-TBK (1:200, CST, China). Cell nuclei were counterstained with DAPI (Beyotime, China) for 5 minutes.

\section{Statistical analysis}

Data are expressed as the mean \pm standard error of the mean (SEM). Analysis was measured with IBM SPSS Statistics 22 software (SPSS Inc., Armonk, New York, USA). Western blot and open field test results were analyzed by one-way analysis of variance (ANOVA) following post hoc multiple comparison; MWT data were analyzed by two-way analysis of variance (ANOVA) following post hoc multiple comparison. $P$ values $<0.05$ were considered significant.

\section{Results}

\section{Spinal nerve ligation led to severe mechanical hypersensitivity, anxiety and STING/TBK activation}

Our data confirmed that spinal nerve ligation resulted in mechanical hypersensitivity. A significant decrease of MWT was observed at postoperative day $2,4,6$. Since anxiety is a frequent co-morbidity of neuropathic pain, anxiety level was evaluated with OFT. Our data demonstrated that the distance, the proportion of time spent in the center zone were significant decreased in compared with sham group. Representative heat maps and travel traces were shown in Figure 2. Expressions of related proteins were evaluated with Western blotting tests. The up-regulation of LC3, FAM134b, Grp78 and down-regulation of p62 confirmed that SNL induced ER-phagy impairment and ER stress. Furthermore, p-STING/STING and $\mathrm{p}$-TBK/TBK was increased in SNL group compared with sham group, indicating STING/TBK pathway was activated in SNL rats.

\section{Distribution and cellular localization of p-TBK and Grp78 in SNL rats}

In this study, we evaluated the distribution of STING/TBK and ER stress in SNL rats. Our data demonstrated that p-TBK mainly expressed in astrocytes of lamina III-IV in SNL rats (Figure 3A, B). Furthermore, ER stress marker, Grp78, mainly expressed in neurons of lamina I-IV (Figure 3C, D). Our data suggested that STING/TBK pathway in astrocytes was activated following SNL induced ER stress in neurons. 


\section{Dexmedetomidine neutralized SNL induced nociception, anxiety and STING/TBK pathway activation}

Dexmedetomidine administration resulted in significant analgesic and antianxiety effects. In $S N L+D$ group, MWT was increased at postoperative day 4, 6 compared with SNL group (Figure 4A). Dexmedetomidine injection significant increased the proportion of time spent in the center zone compared with SNL group (Figure 4B). p-STING, p-TBK was significantly decreased indicating STING/TBK pathway was down-regulated following dexmedetomidine administration. Up-regulation of FAM134b and down-regulation of LC3, p62, Grp78 suggested ER-phagy level was enhanced and ER stress was attenuated. Moreover, 2'3'-cGAMP administration resulted in reversed the analgesic and antianxiety effects induced by dexmedetomidine administration (Figure 4A, B). In SNL+D+G group, p-STING, p-TBK, FAM134b, LC3, p62, Grp78 were increased compared with SNL+D group, indicating 2'3'-cGAMP administration induced ER-phagy impairment and ER stress via activating STING/TBK pathway (Figure 4D). Representative heat maps and travel traces of con group, $S N L$ group, $S N L+D, S N L+D+G$ group were shown in Figure 4C.

\section{Ketamine neutralized SNL induced nociception, anxiety and STING/TBK pathway activation}

Ketamine administration led to significant analgesic and antianxiety effects. In SNL+K group, MWT was increased at postoperative day 2, 4, 6 compared with SNL+V group. Ketamine injection significant increased that the distance and the proportion of time spent in the center zone compared with SNL group. Representative heat maps and travel traces were shown in Figure 5. Ketamine administration decreased the expression level of p-STING, p-TBK, p62, Grp78 and increased the expression level of FAM134b, LC3 compared with SNL group. Data suggested that ketamine ameliorated ER stress via increasing ER-phagy through inhibiting STING/TBK pathway. Furthermore, 2'3'-cGAMP neutralized the analgesic and antianxiety effects induced following ketamine administration assayed using MWT and OFT. Moreover, in $\mathrm{SNL}+\mathrm{K}+\mathrm{G}$ group, the protein expression level of p-STING, p-TBK, FAM134b, p62, Grp78 was increased and the expression level of LC3 was decreased compared with SNL+K group. Data revealed that 2'3'-cGAMP activated STING/TBK pathway and resulted in blocking the degradation of ER-phagy to enhance ER stress.

\section{Discussion}

In this study, our results showed that SNL induced significant mechanical allodynia and anxiety in rats, furthermore, ER stress and ER-phagy impairment via STING pathway activation. The mechanical allodynia and anxiety in SNL rats can be attenuated by injection of dexmedetomidine and ketamine respectively. Of note, both dexmedetomidine and ketamine relieved allodynia and anxiety induced by SNL via down-regulating STING/TBK pathway to ameliorate spinal ER stress. However, ER-phagy levels were altered differently following dexmedetomidine and ketamine treatment respectively. 
Dexmedetomidine and ketamine produced analgesic and anxiolytic effects as premedication before general anesthesia(32,33). Furthermore, administration of dexmedetomidine and ketamine both could reverse central sensitization induced by neuropathic pain $(8,34)$. In this study, our data demonstrated that dexmedetomidine and ketamine served antiallodynic and anxiolytic effects in animal neuropathic pain condition $(9,35-37)$, suggesting dexmedetomidine and ketamine might be promising pharmacotherapies.

Dexmedetomidine is a highly selective a-2 agonist, its affinity for a-2 AR is greater compared with clonidine $(35,38)$, which could enhancing norepinephrine (NE) concentration in the spinal cord of neuropathic pain patients together with amitriptyline and duloxetine. In addition, treatments such as amitriptyline and duloxetine are regard as first-line pharmacotherapies for neuropathic pain in clinical guideline nowadays $(39,40)$. In this case, dexmedetomidine might be widespread used as neuropathic pain treatment in the future. However, little is known about the underlying molecular mechanisms in which dexmedetomidine attenuates neuropathic pain induced allodynia and anxiety.

Studies focus on the neurotoxicity of high dosing ketamine administration and long-term ketamine abuse $(41,42)$. However, ketamine served as proper rescue, pharmacotherapy and, also, adjuvant for neuropathic pain with promising application prospect(8). Sub-anesthetic dosing of Ketamine provided clinical and experimental benefits for chronic pain and depression $(43,44)$. Since it is so, it is essential to investigate the mechanisims of ketamine administration in neuropathic pain model.

Out data demonstrated that dexmedetomidine and ketamine reversed SNL induced allodynia and anxiety significantly. As shown in Figure 4, 5, mechanical allodynia was attenuated in SNL+D group and SNL+K group compared with SNL group respectively. Dexmedetomidine and ketamine administration induced anxiolytic effect measured with open filed tests. To be specific, dexmedetomidine improved the behavior tests in the proportion of time spent in the center. On the other hand, ketamine improved SNL-rats behaviors in the distance and the proportion of time spent in the center. Our data suggested that ketamine could both improve the performance of locomotion and antianxiety. In addition, dexmedetomidine and ketamine both provided a satisfactory analgesic effect in SNL induced neuropathic pain rats.

To determine whether ER-phagy was modulated following dexmedetomidine and ketamine administration in SNL rats, the expression of FAM134b, LC3 and p62 were measured with immunoblotting. Interestingly, our data revealed ER-phagy processing were modulated differently following dexmedetomidine and ketamine administration, respectively.

Dexmeditomidine treatment resulted in FAM134b significant increased, LC3 and p62 decreased significantly, indicating the level of ER bind to autophagosomes and ER degradation by ER-phagy was increased, however, the autophagosome level was decreased. The autophagosome decreased might result from the unbalance of formation and effective degradation of autophagosome.

Ketamine treatment induced FAM134b, LC3 significant increased and p62 significant decreased, suggesting spinal ER-phagy significant increased. Data suggested that more ER was selectively removed 
by autophgosomes and degradation by ER-phagy processing. Dexmedetomidine rendered the degradation of ER-phagy increase, and ketamine induced both the formation and degradation of autophagosomes swallowed ER increased. Even so, both dexmedetomidine and ketamine administration led to enhance ER-phagy process. Moreover, 2'3'-cGAMP administration induced LC3 increased in $S N L+D+G$ group compared with $S N L+D$ group, indicating autophagosome level being increased following 2'3'-cGAMP injection. On the other hand, 2'3'-cGAMP decreased the expression level of LC3 in SNL+K+G group compared with SNL+K group.

Recently studies demonstrated that STING pathway had tremendous potential for immunotherapy from bench to bedside(24, 45-47). However, whether STING pathway involved in neuropathic pain process and whether STING pathway regulate ER-phagy process still remain unknown. In this study, our data indicated that STING/TBK pathway was activated in the spinal cord of neuropathic pain model.

Activated STING signaling triggers ER stress(48). STING-containing endoplasmic reticulum-Golgi intermediate compartment (ERGIC) served as a membrane source for the lipidation of $\operatorname{LC} 3(20,49,50)$. STING directly interacted with LC3 and regulated autophagy process(19). Thus, STING modulates autophagy, an essential mechanism to keep homeostasis following ER stress, via localizing to autophagosomes $(15,17,51,52)$. Specifically, Studies suggested that altered STING pathway driving ERphagy to resolves ER stress $(21,53,54)$. Our data revealed that dexmedetomidine and ketamine upregulated the level of ER-phagy via inhibiting STING/TBK pathway. Moreover, activating STING pathway via 2'3'-cGAMP resulted in neutralizing the treatment effects of dexmedetomidine and ketamine induced through impairing ER-phagy. We investigated the distribution and cellular localization of spinal STING/TBK and ER stress using immunofluorescence. p-TBK mainly expressed in astrocytes, Grp78 mainly expressed in neurons, indicating that SNL induced spinal ER stress in neurons through activating spinal STING pathway in astrocytes. These results expanded our understanding of the interaction of neurons and astrocytes in the spinal cord of SNL rats.

However, this study failed to demonstrate whether dexmedetomidine and ketamine provide doesdependent analegic and antianxiety effects in SNL rats. Furthermore, STING pathway might be involved in immune response, the crosstalk between ER-phagy and immune response in neurons or glial cells still needs further researches to illustrate.

\section{Conclusions}

STING/TBK signaling pathway in astrocytes was activated in the spinal cord of SNL rats. Our data suggested that both dexmedetomidine and ketamine could elevate the level of ER-phagy via inhibiting STING pathway to attenuate ER stress in SNL rats. Nevertheless, dexmedetomidine and ketamine raised the level of spinal ER-phagy in SNL rats differently. 2'3'-cGAMP elevated STING pathway to attenuate dexmedetomidine and ketamine treatment effects via modulating ER-phagy.

\section{Abbreviations}


STING: stimulator of interferon genes

mPFC: medial prefrontal cortex

SNL: Spinal nerve ligation

MWT: mechanical withdrawal threshold

OFT: open field test

AR: selective a2 adrenergic receptor

ER: endoplasmic reticulum

TBK: TANK binding kinase

IRF3: interferon regulatory factor3

SD: Sprague Dawley

ANOVA: analysis of variance

NE: norepinephrine

ERGIC: endoplasmic reticulum-Golgi intermediate compartment

\section{Declarations}

Ethics approval consent to participate

All procedures were approved by the Animal Ethics Care and Use Committee of China Medical University Shengjing Hospital (approved number冈2016PS013K).

\section{Consent of publication}

Not applicable

\section{Availability of data and materials}

All data generated or analysed during this study are included in this published article

\section{Competing interests}

None.

\section{Funding}


This work is supported by grants from the National Natural Science Foundation of China (No.81671311, No.81870838), the Key Research and Development Program of Liaoning Province (No.2018225004) and the Outstanding Scientific Fund of Shengjing Hospital (No. 201708).

\section{Authors' contributors}

Yongda Liu designed the study and wrote the manuscript. Jiao Guo, Xingyue Li, Guang Han analyzed data and revised the manuscript. Yongda Liu, Zhibin Wang, Mengmeng Ding performed the research. Ping Zhao supervised the study.

\section{Acknowledgements}

All the authors would like to thank Lili Wang and Jianing Miao for technical support.

\section{References}

1. Costigan M, Scholz J, Woolf CJ. Neuropathic pain: a maladaptive response of the nervous system to damage. Annual review of neuroscience. 2009;32:1-32.

2. Scholz J, Woolf CJ. The neuropathic pain triad: neurons, immune cells and glia. Nature neuroscience. 2007;10(11):1361-8.

3. Tsuda M, Koga K, Chen T, Zhuo M. Neuronal and microglial mechanisms for neuropathic pain in the spinal dorsal horn and anterior cingulate cortex. J Neurochem. 2017;141(4):486-98.

4. Sdrulla AD, Guan Y, Raja SN. Spinal Cord Stimulation: Clinical Efficacy and Potential Mechanisms. Pain Pract. 2018;18(8):1048-67.

5. Lin JP, Chen CQ, Huang LE, Li NN, Yang Y, Zhu SM, et al. Dexmedetomidine Attenuates Neuropathic Pain by Inhibiting P2X7R Expression and ERK Phosphorylation in Rats. Exp Neurobiol. 2018;27(4):267-76.

6. Avula RR, Vemuri NN, Puthi S. Ultrasound-Guided Subclavian Perivascular Brachial Plexus Block Using 0.5\% Bupivacaine with Dexmedetomidine as an Adjuvant: A Prospective Randomized Controlled Trial. Anesth Essays Res. 2019;13(4):615-9.

7. Gao Z, Xiao Y, Wang Q, Li Y. Comparison of dexmedetomidine and dexamethasone as adjuvant for ropivacaine in ultrasound-guided erector spinae plane block for video-assisted thoracoscopic lobectomy surgery: a randomized, double-blind, placebo-controlled trial. Ann Transl Med. 2019;7(22):668.

8. Orhurhu V, Orhurhu MS, Bhatia A, Cohen SP. Ketamine Infusions for Chronic Pain: A Systematic Review and Meta-analysis of Randomized Controlled Trials. Anesth Analg. 2019;129(1):241-54.

9. Fang X, Zhan G, Zhang J, Xu H, Zhu B, Hu Y, et al. Abnormalities in Inflammatory Cytokines Confer Susceptible to Chronic Neuropathic Pain-related Anhedonia in a Rat Model of Spared Nerve Injury. Clin Psychopharmacol Neurosci. 2019;17(2):189-99. 
10. Ge Y, Jiao Y, Li P, Xiang Z, Li Z, Wang L, et al. Coregulation of endoplasmic reticulum stress and oxidative stress in neuropathic pain and disinhibition of the spinal nociceptive circuitry. Pain. 2018;159(5):894-906.

11. Liu X, Zhu M, Ju Y, Li A, Sun X. Autophagy dysfunction in neuropathic pain. Neuropeptides. 2019;75:41-8.

12. Marinelli S, Nazio F, Tinari A, Ciarlo L, D'Amelio M, Pieroni L, et al. Schwann cell autophagy counteracts the onset and chronification of neuropathic pain. Pain. 2014;155(1):93-107.

13. Zhang E, Yi MH, Ko Y, Kim HW, Seo JH, Lee YH, et al. Expression of LC3 and Beclin 1 in the spinal dorsal horn following spinal nerve ligation-induced neuropathic pain. Brain research. 2013;1519:31-9.

14. Sisinni L, Pietrafesa M, Lepore S, Maddalena F, Condelli V, Esposito F, et al. Endoplasmic Reticulum Stress and Unfolded Protein Response in Breast Cancer: The Balance between Apoptosis and Autophagy and Its Role in Drug Resistance. Int J Mol Sci. 2019;20(4).

15. Senft D, Ronai ZA. UPR, autophagy, and mitochondria crosstalk underlies the ER stress response. Trends Biochem Sci. 2015;40(3):141-8.

16. Chino H, Hatta T, Natsume T, Mizushima N. Intrinsically Disordered Protein TEX264 Mediates ERphagy. Mol Cell. 2019;74(5):909-21 e6.

17. Grumati P, Dikic I, Stolz A. ER-phagy at a glance. J Cell Sci. 2018;131(17).

18. Loi M, Fregno I, Guerra C, Molinari M. Eat it right: ER-phagy and recovER-phagy. Biochem Soc Trans. 2018;46(3):699-706.

19. Gui X, Yang H, Li T, Tan X, Shi P, Li M, et al. Autophagy induction via STING trafficking is a primordial function of the cGAS pathway. Nature. 2019;567(7747):262-6.

20. Yang Y, Ma F, Liu Z, Su Q, Liu Y, Liu Z, et al. The ER-localized Ca(2+)-binding protein calreticulin couples ER stress to autophagy by associating with microtubule-associated protein 1A/1B light chain 3. J Biol Chem. 2019;294(3):772-82.

21. Moretti J, Roy S, Bozec D, Martinez J, Chapman JR, Ueberheide B, et al. STING Senses Microbial Viability to Orchestrate Stress-Mediated Autophagy of the Endoplasmic Reticulum. Cell. 2017;171(4):809-23 e13.

22. Petrasek J, Iracheta-Vellve A, Csak T, Satishchandran A, Kodys K, Kurt-Jones EA, et al. STING-IRF3 pathway links endoplasmic reticulum stress with hepatocyte apoptosis in early alcoholic liver disease. Proc Natl Acad Sci U S A. 2013;110(41):16544-9.

23. Bai J, Liu F. The cGAS-cGAMP-STING Pathway: A Molecular Link Between Immunity and Metabolism. Diabetes. 2019;68(6):1099-108.

24. Chon HJ, Kim H, Noh JH, Yang H, Lee WS, Kong SJ, et al. STING signaling is a potential immunotherapeutic target in colorectal cancer. J Cancer. 2019;10(20):4932-8.

25. Mathur V, Burai R, Vest RT, Bonanno LN, Lehallier B, Zardeneta ME, et al. Activation of the STINGDependent Type I Interferon Response Reduces Microglial Reactivity and Neuroinflammation. Neuron. 2017;96(6):1290-302 e6. 
26. Fermaintt CS, Sano K, Liu Z, Ishii N, Seino J, Dobbs N, et al. A bioactive mammalian disaccharide associated with autoimmunity activates STING-TBK1-dependent immune response. Nat Commun. 2019;10(1):2377.

27. Liu YD, Wang ZB, Han G, Jin L, Zhao P. Hyperbaric oxygen relieves neuropathic pain through AKT/TSC2/mTOR pathway activity to induce autophagy. J Pain Res. 2019;12:443-51.

28. Liu YD, Wang ZB, Han G, Zhao P. Hyperbaric oxygen treatment attenuates neuropathic pain by elevating autophagy flux via inhibiting

mTOR pathway. Am J Transl Res. 2017;9(5):2629-38.

29. Kim SH, Chung JM. An experimental model for peripheral neuropathy produced by segmental spinal nerve ligation in the rat. Pain. 1992;50(3):355-63.

30. Zhou TT, Wu JR, Chen ZY, Liu ZX, Miao B. Effects of dexmedetomidine on P2X4Rs, p38-MAPK and BDNF in spinal microglia in rats with spared nerve injury. Brain research. 2014;1568:21-30.

31. Kontinen V, Kauppila T, Paananen S, A P, Kalso E. Behavioural measures of depression and anxiety in rats with spinal nerve ligation-induced neuropathy. Pain. 1999;80(1-2):341-6.

32. Turktan M, Yilmaz MB, Hatipoglu Z, Ilgaz S, Barc ED, Oksuz H, et al. Molecular determinants of behavioral changes induced by neonatal ketamine and dexmedetomidine application. J Neural Transm (Vienna). 2019;126(12):1577-88.

33. Oriby ME. Comparison of Intranasal Dexmedetomidine and Oral Ketamine Versus Intranasal Midazolam Premedication for Children Undergoing Dental Rehabilitation. Anesth Pain Med. 2019;9(1):e85227.

34. Xun S, Zheng R. Dexmedetomidine alleviates neuropathic pain by regulating JAK/STAT pathway in rats. J Cell Biochem. 2020;121(3):2277-83.

35. Qian Y, Wang Q, Jiao J, Wang G, Gu Z, Huang D, et al. Intrathecal injection of dexmedetomidine ameliorates chronic neuropathic pain via the modulation of MPK3/ERK1/2 in a mouse model of chronic neuropathic pain. Neurol Res. 2019;41(12):1059-68.

36. Huang X, Deng R, Tu W, Hu Z. Dexmedetomidine reduces neuropathic pain in a rat model of skin/muscle incision and retraction. Asian J Surg. 2017;40(1):35-40.

37. Liang F, Liu M, Fu X, Zhou X, Chen P, Han F. Dexmedetomidine attenuates neuropathic pain in chronic constriction injury by suppressing NR2B, NF-kappaB, and iNOS activation. Saudi Pharm J. 2017;25(4):649-54.

38. Yang Y, Xia Z, Meng Q, Liu K, Xiao Y, Shi L. Dexmedetomidine relieves neuropathic pain by inhibiting hyperpolarization-activated cyclic nucleotide-gated currents in dorsal root ganglia neurons. Neuroreport. 2018;29(12):1001-6.

39. Attal N. Pharmacological treatments of neuropathic pain: The latest recommendations. Rev Neurol (Paris). 2019;175(1-2):46-50. 
40. Kremer M, Yalcin I, Goumon Y, Wurtz X, Nexon L, Daniel D, et al. A Dual Noradrenergic Mechanism for the Relief of Neuropathic Allodynia by the Antidepressant Drugs Duloxetine and Amitriptyline. The Journal of Neuroscience. 2018;38(46):9934-54.

41. Meng C, Yao XQ, Chang RJ, Wang SL, Wang X, Ma DQ, et al. Exogenous GM1 Ganglioside Attenuates Ketamine-Induced Neurocognitive Impairment in the Developing Rat Brain. Anesth Analg. 2020;130(2):505-17.

42. Li Y, Ding R, Ren X, Wen G, Dong Z, Yao H, et al. Long-term ketamine administration causes Tau protein phosphorylation and Tau protein-dependent AMPA receptor reduction in the hippocampus of mice. Toxicol Lett. 2019;315:107-15.

43. Humo M, Ayazgok B, Becker LJ, Waltisperger E, Rantamaki T, Yalcin I. Ketamine induces rapid and sustained antidepressant-like effects in chronic pain induced depression: Role of MAPK signaling pathway. Prog Neuropsychopharmacol Biol Psychiatry. 2020:109898.

44. Yang $Y$, Song $Y$, Zhang $X$, Zhao W, Ma T, Liu Y, et al. Ketamine relieves depression-like behaviors induced by chronic postsurgical pain in rats through anti-inflammatory, anti-oxidant effects and regulating BDNF expression. Psychopharmacology (Berl). 2020.

45. Catalano C, da Silva Filho MI, Frank C, Lu S, Jiraskova K, Vymetalkova V, et al. Epistatic effect of TLR3 and cGAS-STING-IKKepsilon-TBK1-IFN signaling variants on colorectal cancer risk. Cancer Med. 2020;9(4):1473-84.

46. Lian Y, Duffy KJ, Yang J. STING Activation and its Application in Immuno-Oncology. Curr Top Med Chem. 2019;19(24):2205-27.

47. Su T, Zhang Y, Valerie $K$, Wang XY, Lin S, Zhu G. STING activation in cancer immunotherapy. Theranostics. 2019;9(25):7759-71.

48. Wu J, Chen YJ, Dobbs N, Sakai T, Liou J, Miner JJ, et al. STING-mediated disruption of calcium homeostasis chronically activates ER stress and primes T cell death. J Exp Med. 2019;216(4):86783.

49. Larabi A, Barnich N, Nguyen HTT. New insights into the interplay between autophagy, gut microbiota and inflammatory responses in IBD. Autophagy. 2020;16(1):38-51.

50. Dong C, Zheng H, Huang S, You N, Xu J, Ye X, et al. Heme oxygenase-1 enhances autophagy in podocytes as a protective mechanism against high glucose-induced apoptosis. Experimental cell research. 2015;337(2):146-59.

51. Wilkinson S. ER-phagy: shaping up and destressing the endoplasmic reticulum. FEBS J. 2019;286(14):2645-63.

52. Song S, Tan J, Miao Y, Zhang Q. Crosstalk of ER stress-mediated autophagy and ER-phagy: Involvement of UPR and the core autophagy machinery. J Cell Physiol. 2018;233(5):3867-74.

53. Kary C. Liver autophagy's sweet side. Nature cell biology. 2018;20(3):224.

54. Moretti J, Blander JM. Detection of a vita-PAMP STINGs cells into reticulophagy. Autophagy. 2018;14(6):1102-4. 


\section{Figures}

\begin{tabular}{|c|c|c|c|c|c|c|c|c|}
\hline pharmacotherapy & & $\mathrm{D} / \mathrm{K}$ & $\mathrm{D} / \mathrm{K}$ & & $\mathrm{D} / \mathrm{K}$ & & $D / K$ & \\
\hline 2'3'-cGAMP & & $\mathrm{G}$ & $\mathrm{G}$ & & G & & G & \\
\hline operation procedure & & 0 & & & & & & TC \\
\hline behavior test(s) & M & & & $M$ & & M & & $\mathrm{M}+\mathrm{OF}$ \\
\hline
\end{tabular}

\section{Figure 1}

Timeline of experimental procedure Abbreviation: $\mathrm{D}$ : demedetomidine administration; $\mathrm{K}$ : ketamine administration; G: 2'3'-cGAMP administration; O: spinal nerve ligation operation; TC: tissue collection; M: mechanical withdrawal threshold; OF: open field test. 
A

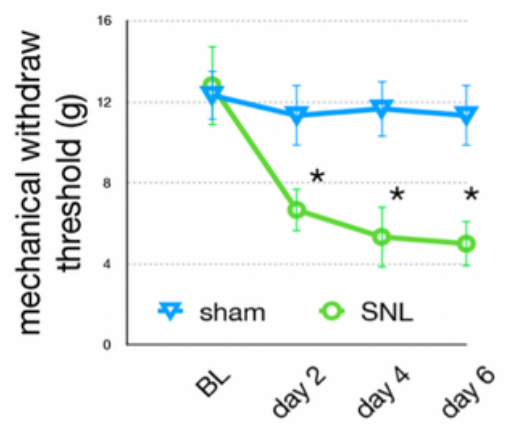

$\mathrm{B}$

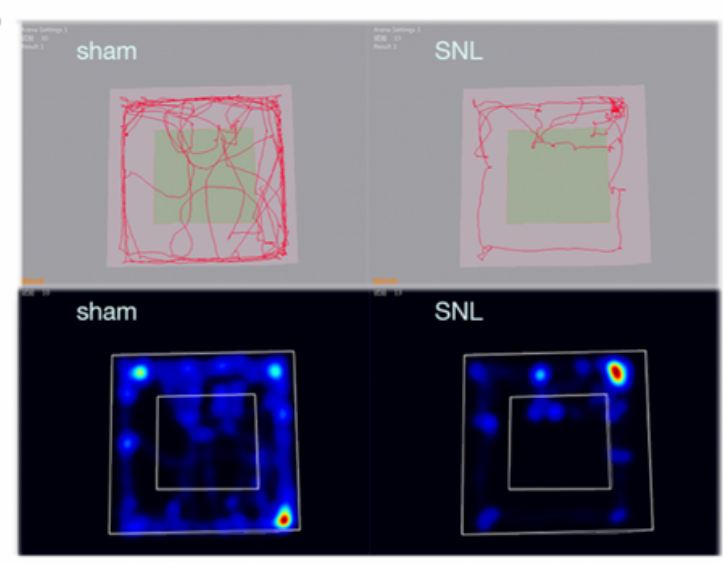

C
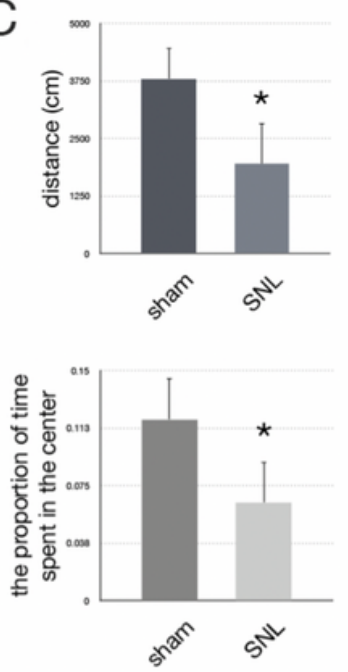

E
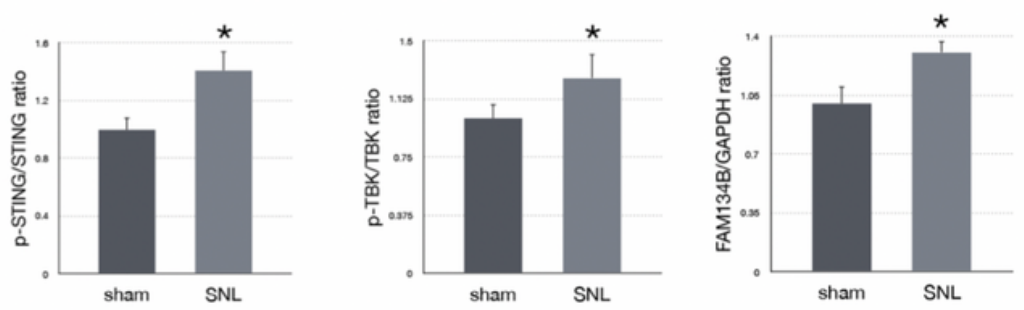

$55 \mathrm{kDa}$

14, 16 kDa

$62 \mathrm{kDa}$

$78 \mathrm{kDa}$

$36 \mathrm{kDa}$
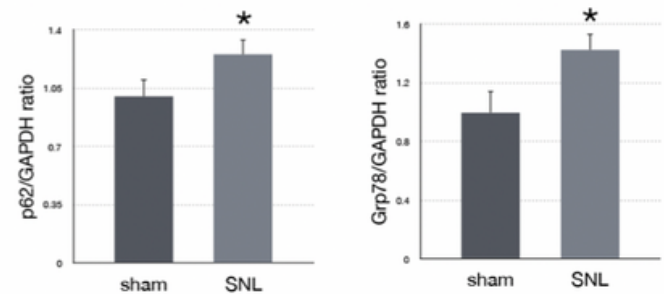

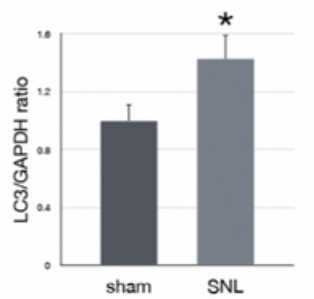

\section{Figure 2}

Spinal nerve ligation activated STING/TBK pathway (A) Mechanical withdrawal threshold in sham group and SNL group (two-way ANOVA). (B) The trace map and heat map of open field test in sham group and SNL group. (C) Quantification of open field test (one-way ANOVA, distance: $F=16.781, p=0.002$; the proportion of time spent in the center: $F=12.512, p=0.005$ ). (D) Western blotting in sham group and $S N L$ group. (E) Quantification of immunoblotting in sham group and SNL group (one-way ANOVA, pSTING/STING: $F=16.75, p=0.002 ; p-T B K / T B K=10.786, p=0.008 ; F A M 134 b / G A P D H: ~ F=19.265, p=0.001$; LC3/GAPDH: $F=10.756, p=0.008 ; p 62 / G A P D H: ~ F=8.957, p=0.014 ;$ Grp78/GAPDH: $F=10.897 . p=0.008$ ). $\mathrm{n}=6$ rats per group, ${ }^{*} \mathrm{p}<0.05$ compared with sham group. Abbreviation: $\mathrm{SNL}$ : spinal nerve ligation; sham: sham operation of spinal nerve ligation; BL: baseline 


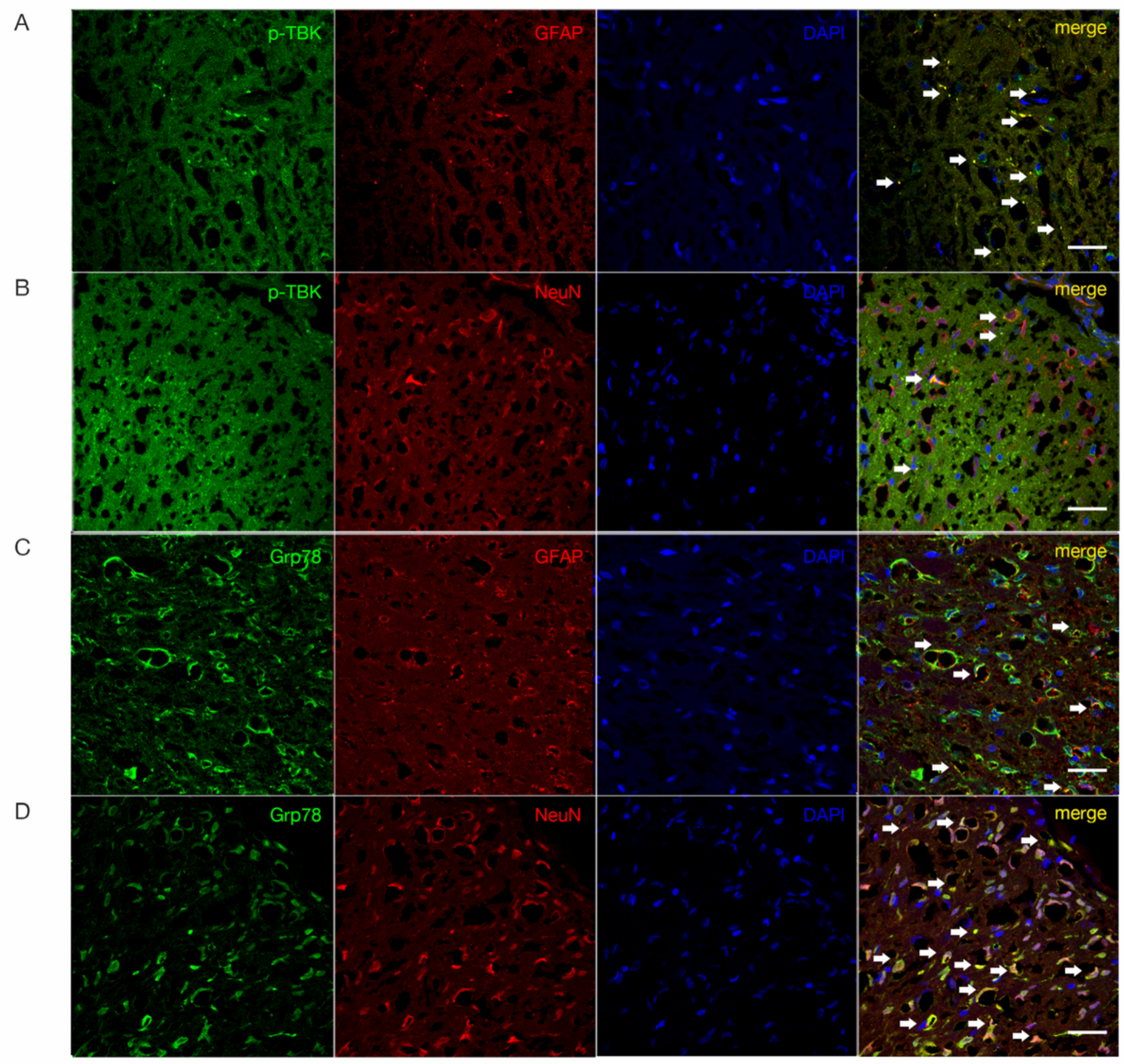

Figure 3

Co-localization of p-TBK/Grp78 with GFAP and NeuN in ipsilateral spinal dorsal horn of SNL rat. (A) Confocal immunofluorescence of p-TBK and GFAP in laminae I-III of SNL rats L5 level. (B) Confocal immunofluorescence of $\mathrm{p}-\mathrm{TBK}$ and NeuN in laminae I-III of SNL rats L5 level. (C) Confocal immunofluorescence of Grp78 and GFAP in laminae I-III of SNL rats L5 level. (D) Confocal immunofluorescence of Grp78 and NeuN in laminae I-III of SNL rats L5 level. n=6 rats per group. Scale bar $=50 \mu \mathrm{m}$. 
A

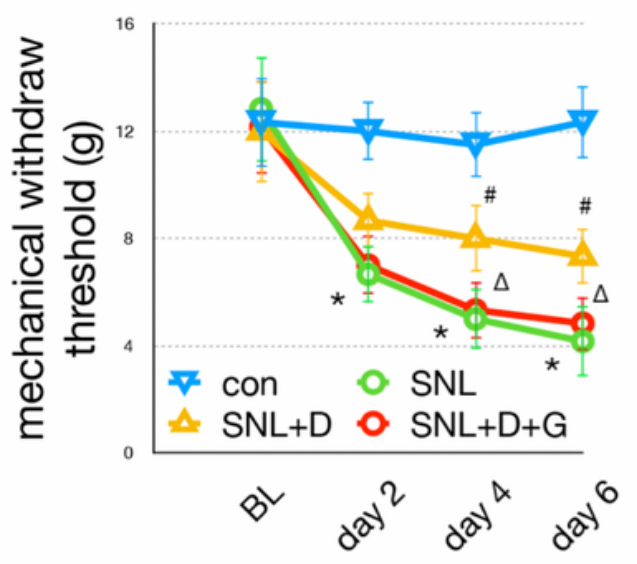

B

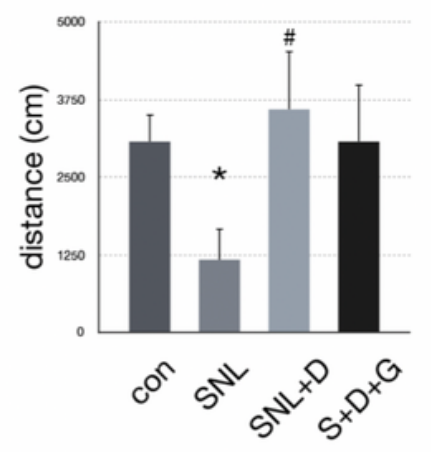

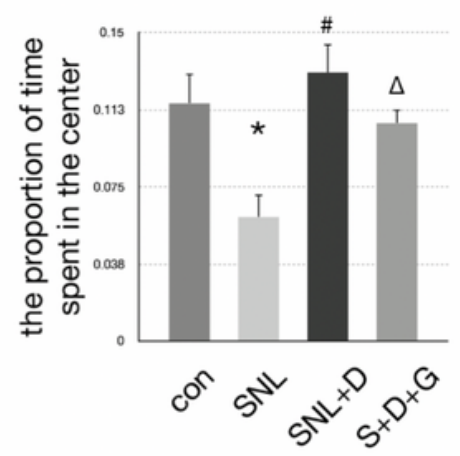

C

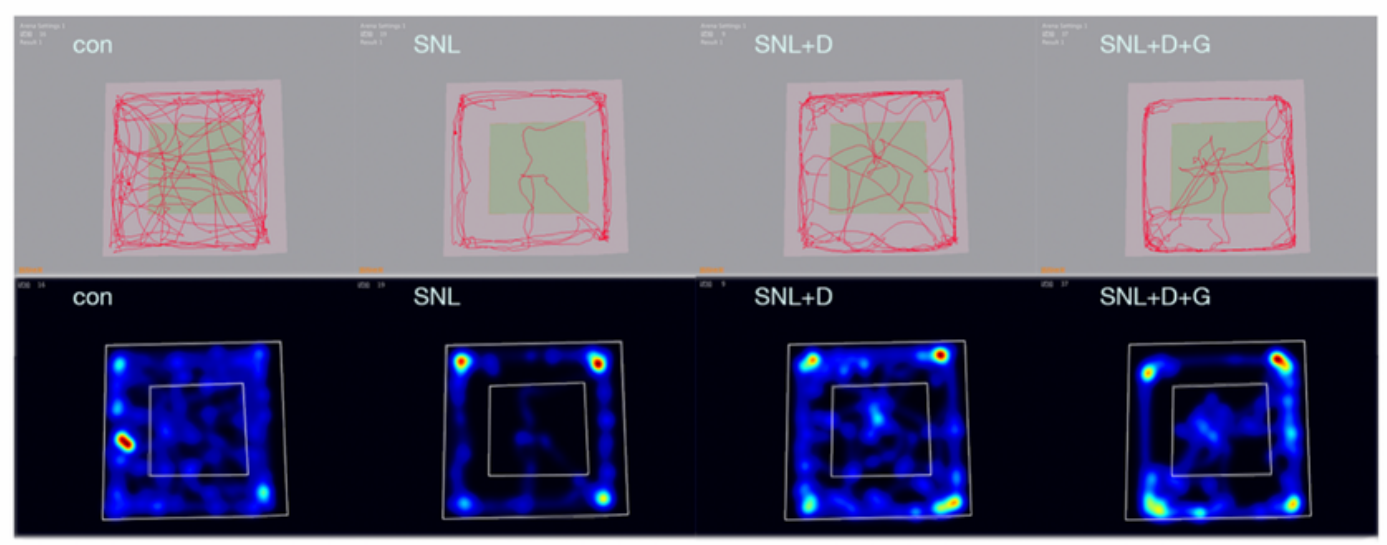

D

SNL
Dex
2'3'-cGAMP
p-STING
p-TBK

FAM134B

LC3

p62

Grp78

GAPDH
$-++$

$-++$

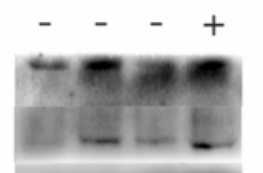

$41 \mathrm{kDa}$

$84 \mathrm{kDa}$

55 kDa

14, 16 kDa

$62 \mathrm{kDa}$

78 kDa

$36 \mathrm{kDa}$

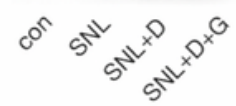

E
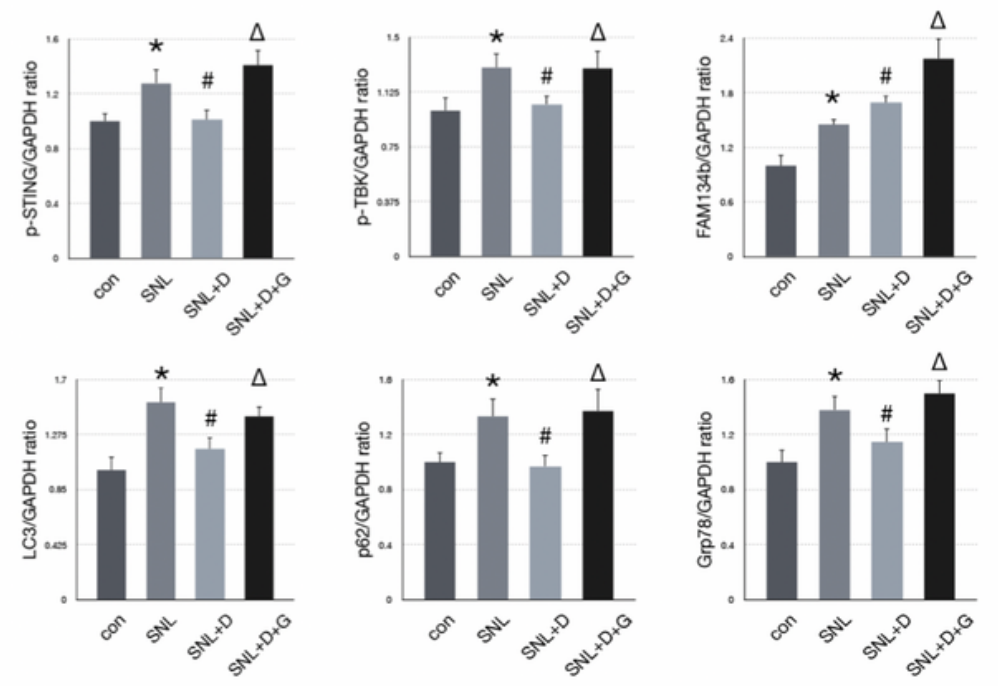

\section{Figure 4}

Dexmedetomidine provided antianxiety effect via inhibiting STING/TBK pathway. (A) MWT tests of con group, SNL group, SNL+D and SNL+D+G group (two-way ANOVA) (B) Quantification of open field of con group, $S N L$ group, $S N L+D$ and $S N L+D+G$ group (one-way ANOVA, distance: $F=13.260, p=0.003$; the proportion of time spent in the center: $F=16.520, p=0.006)$. (C) Trace map and heat map of con group, $S N L$ group, $S N L+D$ and $S N L+D+G$ group. (D) Western blot of con group, $S N L$ group, $S N L+D$ and $S N L+D+G$ 
group. (E) Western blot quantification of con group, $S N L$ group, $S N L+D$ and $S N L+D+G$ group (one-way ANOVA, $p-S T I N G / G A P D H: F=6.092, p=0.006$; $p$-TBK/GAPDH: $F=3.709, p=0.034 ; F A M 134 b / G A P D H:$ $F=13.979, p=0.007$; LC3/GAPDH: $F=9.265, p=0.001 ; p 62 / G A P D H: F=4.268, p=0.022 ; G r p 78 / G A P D H:$ $F=8.613, p=0.001) . n=6$ rats per group, ${ }^{*} p<0.05$ compared with con group; $\# p<0.05$ compared with $S N L$ group; $\Delta p<0.05$ compared with SNL+D group. Abbreviation: BL: baseline; Dex: dexmedetomidine; con group: rats received sham operation, vehicle of dexmedetomidine and 2'3'-cGAMP administration; SNL group: rats received SNL procedure and vehicle of dexmedetomidine and 2'3'-cGAMP administration; $S N L+D$ group: rats received SNL and dexmedetomidine and vehicle of 2'3'-cGAMP. $S N L+D+G$ group: rats received SNL and dexmedetomidine and 2'3'-cGAMP. 
A

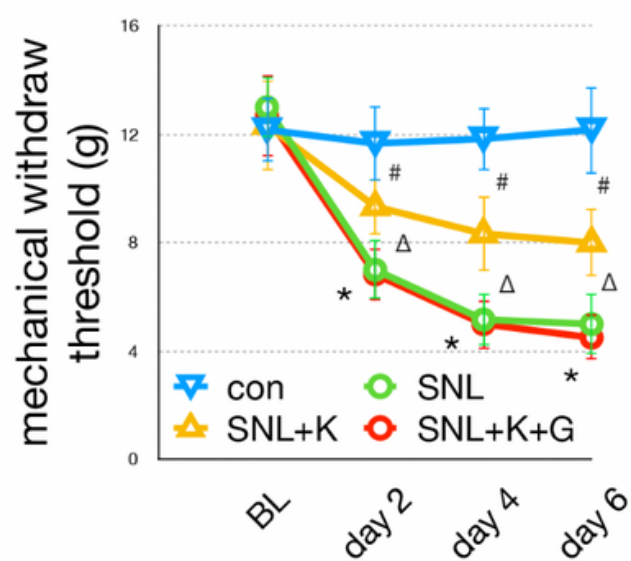

B
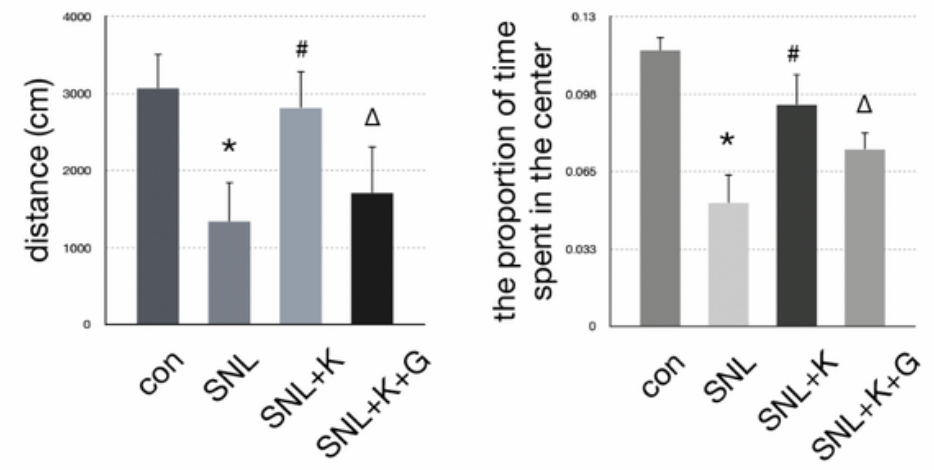

C

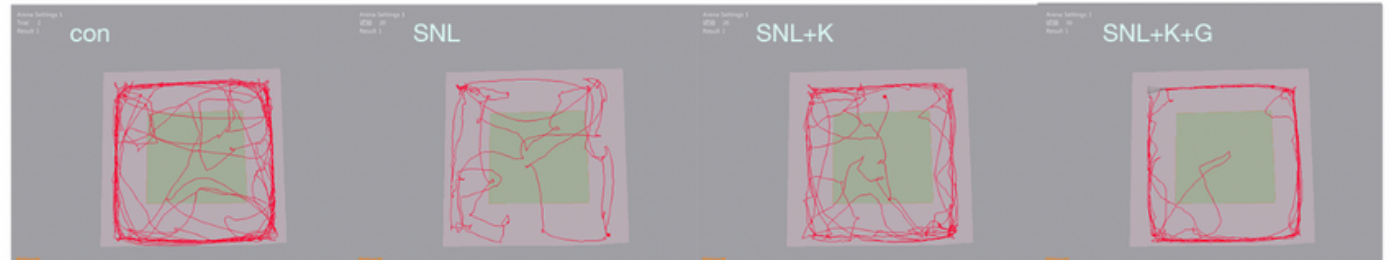

con

SNL

SNL+K

SNL+K+G

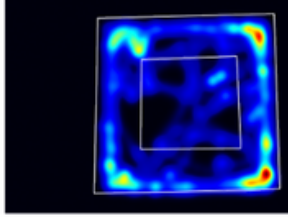

D

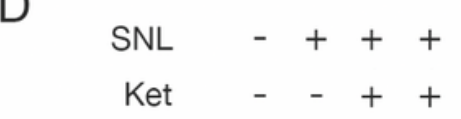

2'3'-cGAMP

p-STING

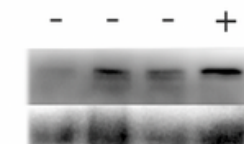

$41 \mathrm{kDa}$

p-TBK

$84 \mathrm{kDa}$

FAM134B

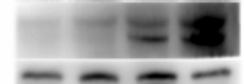

$55 \mathrm{kDa}$

LC3

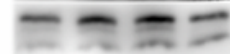

$14,16 \mathrm{kDa}$

p62

$62 \mathrm{kDa}$

Grp78

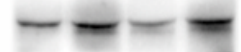

$78 \mathrm{kDa}$

GAPDH

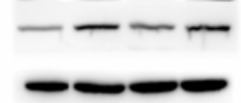

$36 \mathrm{kDa}$

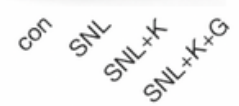

E
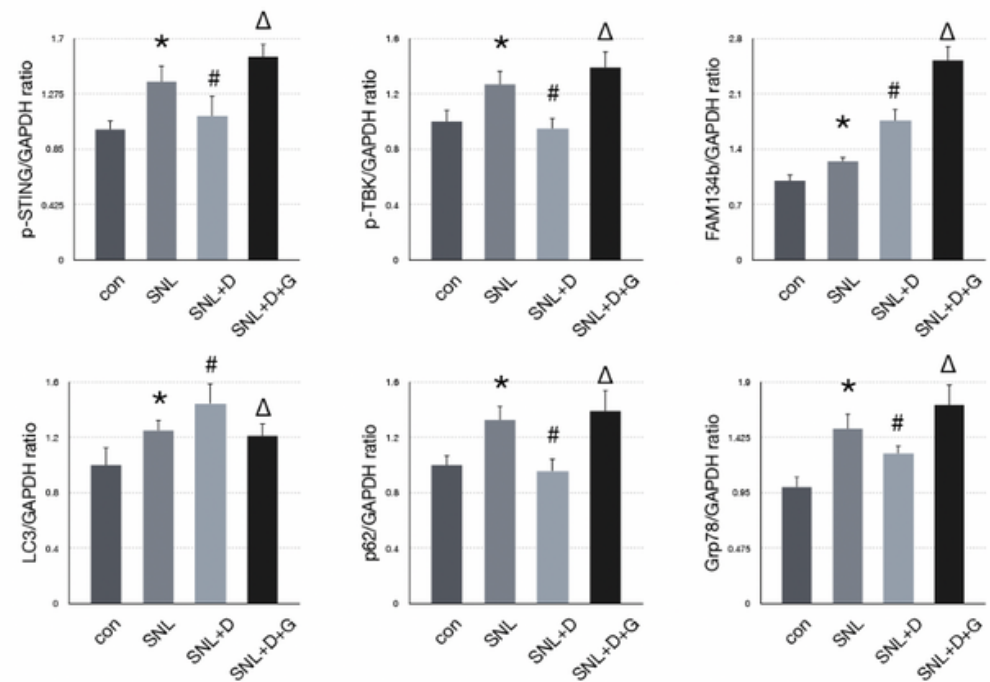

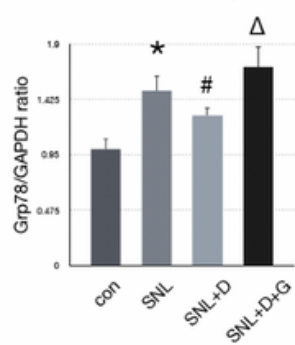

\section{Figure 5}

Ketamine provided antianxiety effect via inhibiting STING/TBK pathway. (A) MWT tests of con group, SNL group, $S N L+K$ and $S N L+K+G$ group (two-way ANOVA) (B) Quantification of open field of con group, $S N L$ group, $S N L+K$ and $S N L+K+G$ group (one-way ANOVA, distance: $F=16.034, p=0.002$; the proportion of time spent in the center: $F=11.637, p=0.005)$. (C) Trace map and heat map of con group, SNL group, $S N L+K$ and $S N L+K+G$ group. (D) Western blot of con group, $S N L$ group, $S N L+K$ and $S N L+K+G$ group. (E) Western blot quantification of con group, $S N L$ group, $S N L+K$ and $S N L+K+G$ group (one-way ANOVA, $p$ - 
STING/GAPDH: $F=10.419, p=0.001$; $p-T B K / G A P D H: F=12.993, p=0.003 ; F A M 134 b / G A P D H: F=16.606$, $p=0.005$; LC3/GAPDH: $F=11.014, p=0.005 ; p 62 / G A P D H: F=4.047, p=0.026 ;$ Grp78/GAPDH: $F=7.520$, $p=0.002) . n=6$ rats per group, ${ }^{*} p<0.05$ compared with con group; $\# p<0.05$ compared with SNL group; $\Delta p<0.05$ compared with SNL+K group. Abbreviation: BL: baseline; Ket: ketamine; con group: rats received sham operation, vehicle of ketamine and 2'3'-cGAMP administration; SNL group: rats received SNL procedure and vehicle of ketamine and 2'3'-cGAMP administration; SNL+K group: rats received SNL and ketamine and vehicle of 2'3'-cGAMP. SNL+K+G group: rats received SNL and ketamine and 2'3'-cGAMP. 